\title{
Plaquette Ordering in SU(4) Antiferromagnets
}

\author{
Anup Mishra, Michael Ma, Fu-Chun Zhang, \\ Department of Physics, \\ University of Cincinnati, Cincinnati, OH 45221-0011
}

(October 31, 2018)

PACS numbers: $75.10 . J m, 11.30 .-\mathrm{j}$

\begin{abstract}
We use fermion mean field theory to study possible plaquette ordering in the antiferromagnetic SU(4) Heisenberg model. We find the ground state for both the square and triangular lattices to be the disconnected plaquette state. Our mean field theory gives a first order transition for plaquette ordering for the triangular lattice. Our results suggest a large number of low lying states.
\end{abstract}


Recently, the importance of orbital degeneracy in the physics of the insulating phase of transition metal oxides has been emphasized [1] 3]. The understanding of the ground state and elementary excitations in these materials requires consideration of both the orbital and spin degrees of freedom. In $\mathrm{V}_{2} \mathrm{O}_{3}$ for example, the anomalous ordering of the antiferromagnetic insulator can be understood quite simply as a result of spin-orbital coupling [4] 8]. The interplay between spins and orbitals may also be responsible for the lack of magnetic ordering down to very low temperature in $\mathrm{LiNiO}_{2}$ [9]12]. The orbital ordering and correlations have recently been observed in synchrotron x-ray diffraction measurements [13] and in resonant x-ray scattering experiments [14].

It has been pointed out that for a $S=1 / 2$ system with a double orbital degeneracy there is an ideal limit where the spin and orbital degrees of freedom can be unified into a higher $\mathrm{SU}(4)$ symmetry [15,3]. While this symmetry is usually not realized in the transition metal oxides due to Hund's rule and anisotropy in hopping amplitudes, the SU(4) symmetric point may still shed light on the physics of more realistic systems, especially if the deviation from the ideal limit is not too large. Using fermion mean field theory(MFT), Li et.al. [3] showed that the $\mathrm{SU}(4)$ ground state on a square lattice possesses no magnetic long range order. Possible candidates for the ground state include the commensurate flux phase $(1 / 4$ flux per plaquette), and the plaquette solid state. The commensurate flux phase has broken time reversal invariance which is troublesome (although time reversal invariance may be restored when projected into the physical Hilbert space of one fermion per site). Simple variational calculations on the square and triangular lattice support the lack of long ranged order, but also suggest the ground state should be a plaquette solid or a resonating plaquette liquid [3]. On the other hand, Schwinger boson mean field theory predicts a long range ordered state [16]. More recently, finite size numerical calculations on the square lattice provides further evidence that the ground state has no magnetic long range order [17]. However, while the commensurate flux phase is two-fold degenerate, their numerical results suggest a 4-fold degeneracy.

A possible explanation for the 4-fold degeneracy is the spontaneous formation of pla- 
quette state with alternating plaquettes of strong and weak correlations. This would be consistent with variational calculations. In this paper, we will investigate plaquette ordering within fermion MFT, a possibility not considered in the mean field calculation of Li et.al. [3]. Using fermion MFT, we will show that the uniform bond amplitude mean field ground state is unstable with respect to plaquette formation on square and triangular lattices. Plaquette ordering breaks the lattice translational invariance. Since the symmetry is discrete, a finite temperature phase transition is possible even in 2 dimensions. Within the MFT, we find the plaquette transition to be first order for the triangular lattice. For the square lattice, the result turns out to be ambiguous.

We start with the antiferromagnetic SU(4) Hamiltonian [3].

$$
H=\sum_{\langle i, j\rangle} \sum_{m, n} S_{m}^{n}(i) S_{n}^{m}(j),
$$

where $\langle i, j\rangle$ are the pairs of the nearest neighbor sites $i$ and $j$ on the lattice, and $m=1,2,3,4$ is the flavor index. $S_{m}^{n}$ are $\mathrm{SU}(4)$ generators. The Hamiltonian is equivalent to an isotropic antiferromagnetic quantum spin-1/2 system with two-fold degenerate orbitals [3]. In terms of electron operators, $S_{m}^{n}(i)=c_{i, m}^{\dagger} c_{i, n}$, where $c_{i, n}$ is the annihilation operator of an electron of flavor $n$ at site $i$, the Hamiltonian in eq.(四) may be rewritten as

$$
H=\sum_{\langle i, j\rangle} \sum_{m, n} c_{i, m}^{\dagger} c_{i, n} c_{j, n}^{\dagger} c_{j, m} .
$$

It is implied that there is a constraint on the number of electrons at every lattice site, given by $\sum_{n} c_{i, n}^{\dagger} c_{i, n}=1$.

In this paper, we shall apply a fermion MFT to study the possibility of plaquette ordering for the square and triangular lattices. Such an ordering would break the translational invariance of the crystal lattice. The fermion MFT is well known from literature [18,19]. We define the bond operator $\Phi_{i j}=\sum_{n} c_{i, n}^{\dagger} c_{j, n}$, and introduce Lagrange multipliers $\lambda_{i}$ to describe the constraint on site $i$. We then use mean field to approximate the bond operator by its self-consistently calculated average, and assume that $\left\langle\Phi_{i j}^{\dagger}\right\rangle=\left\langle\Phi_{i j}\right\rangle=t_{i j}$ is real. Also, 
we replace the local constraint by a global one and set $\lambda_{i}=\lambda$, independent of the site. The mean field Hamiltonian is then given by,

$$
H_{m f}=-\sum_{\langle i, j\rangle, n} t_{i j}\left(c_{i, n}^{\dagger} c_{j, n}+h . c\right)+\sum_{\langle i, j\rangle} t_{i j}^{2}-\lambda\left(\sum_{i, n} c_{i, n}^{\dagger} c_{i, n}-N\right)
$$

where $N$ is the total number of lattice sites, and an overall constant has been dropped for simplicity. We see that $\lambda$ acts as a chemical potential.

We first discuss the square lattice case. As shown in Fig.(1), we consider two types of the bonds in the lattice, denoted by type-A and type-B, representing stronger and weaker bonds, respectively. In a square lattice, there are equal numbers of type- $\mathrm{A}$ and type-B bonds. The hopping amplitude on each bond is thus given by

$$
\begin{aligned}
t_{i j} & =t_{a}, \text { if }\langle i, j\rangle \in\{A\} \\
& =t_{b}, \text { if }\langle i, j\rangle \in\{B\}
\end{aligned}
$$

The mean field Hamiltonian becomes

$$
\begin{aligned}
H_{m f}= & -t \sum_{\vec{k}, n}\left(\cos k_{x} a+\cos k_{y} a\right) c_{\vec{k}, n}^{\dagger} c_{\vec{k}, n} \\
& +i r t \sum_{\vec{k}, n}\left(\sin k_{x} a\right) c_{\vec{k}+(\pi, 0), n}^{\dagger} c_{\vec{k}, n} \\
& +i r t \sum_{\vec{k}, n}\left(\sin k_{y} a\right) c_{\vec{k}+(0, \pi), n}^{\dagger} c_{\vec{k}, n} \\
& +\frac{N t^{2}}{2}\left(1+r^{2}\right)-\lambda\left(\sum_{\vec{k}, n} c_{\vec{k}, n}^{\dagger} c_{\vec{k}, n}-N\right)
\end{aligned}
$$

In the above eqn., $t=t_{a}+t_{b}, r=\left(t_{a}-t_{b}\right) /\left(t_{a}+t_{b}\right), \vec{k}$ is a crystal momentum, and the sum is over the first Brillouin zone.

In a square lattice, the Hamiltonian is invariant under each of the following transformations: a) $t \rightarrow-t$, i.e. $\left(t_{a}, t_{b}\right) \rightarrow\left(-t_{a},-t_{b}\right)$; b) $r \rightarrow-r$, i.e. $\left(t_{a}, t_{b}\right) \rightarrow\left(t_{b}, t_{a}\right)$; and c) $r \rightarrow 1 / r$, i.e. $\left(t_{a}, t_{b}\right) \rightarrow\left(t_{a},-t_{b}\right)$. Therefore, it suffices to consider the parameter space $t \geq 0$, and $0 \leq r \leq 1$. The case of $r=0$ corresponds to the uniform bond amplitude mean-field state and the case of $r=1$ corresponds to a periodic array of disconnected plaquettes. 
The mean field Hamiltonian can be diagonalized and we obtain

$$
H_{m f}=\sum_{\vec{k}, \alpha, n} \epsilon_{\vec{k}, \alpha, n} \beta_{\vec{k}, \alpha, n}^{\dagger} \beta_{\vec{k}, \alpha, n}+\frac{N t^{2}}{2}\left(1+r^{2}\right)-\lambda\left(\sum_{\vec{k}, \alpha, n} \beta_{\vec{k}, \alpha, n}^{\dagger} \beta_{\vec{k}, \alpha, n}-N\right)
$$

where $\beta_{\vec{k}, \alpha, n}^{\dagger}$ is the creation operator for a particle in the state $|\vec{k}, \alpha, n\rangle$ with the energy $\epsilon_{\vec{k}, \alpha, n}$. Here, $\alpha=1,2,3,4$ is the band index, and the sum over $\vec{k}$ is restricted to within the reduced Brillouin zone, which is a quarter of the first Brillouin zone of the crystal. The reduction of the Brillouin zone is the result of the translational symmetry broken by the plaquette state. The four flavor states for a given $\vec{k}$ and $\alpha$ are energetically degenerate. Numerically, we find that for $r \gtrsim 0.3$, the four energy bands are separated by band gaps. The ground state of the system is a filled Fermi sea, with Fermi energy $\mu_{f}=\lambda$ determined by the electron number equation,

$$
\sum_{\vec{k}, \alpha, n}\left\langle\beta_{\vec{k}, \alpha, n}^{\dagger} \beta_{\vec{k}, \alpha, n}\right\rangle=\sum_{\vec{k}, \alpha, n} \theta\left(\lambda-\epsilon_{\vec{k}, \alpha, n}\right)=N
$$

To obtain the mean field ground state of the system, we minimize the energy $E$ below with respect to $t$ and $r$,

$$
\frac{E}{N}=\frac{1}{N} \sum_{\vec{k}, \alpha, n} \epsilon_{\vec{k}, \alpha, n} \theta\left(\lambda-\epsilon_{\vec{k}, \alpha, n}\right)+\frac{t^{2}}{2}\left(1+r^{2}\right) .
$$

We first minimize $E$ with respect to $t$ for a given $r$. The energy as a function of $r$ thus obtained is plotted in Fig.(2). As shown in Fig.(2), the uniform bond amplitude state, given by $r=0$, i.e., $t_{a}=t_{b}$, is locally unstable with respect to the plaquette formation. The energy decreases monotonically as $r$ increases from 0 to 1 . The lowest energy state corresponds to $r=1$, the disconnected plaquette state. The local stability of $r=1$ state can be confirmed independently by solving the weakly connected plaquette problem using simple second order perturbation.

The plaquette state is 4 -fold degenerate. The discrete symmetry implies the ordering will remain stable at low temperature in $2 \mathrm{D}$ and vanishes either continuously or through a first order transition at some finite critical temperature. Within MFT, there will be another 
critical temperature above which $t$ vanishes. Since $t$ represents short-ranged order, this transition is unphysical and is an artifact of this type of MFT.

At a finite temperature, the mean field free energy is given by

$$
\frac{F}{N}=-\frac{k_{b} T}{N} \sum_{\vec{k}, \alpha, n} \ln \left[1+e^{-\beta\left(\epsilon_{\vec{k}, \alpha, n}-\lambda\right)}\right]+\lambda+\frac{t^{2}}{2}\left(1+r^{2}\right),
$$

where $\beta=1 / k_{B} T$, and $\lambda$ is determined by the electron number eq.,

$$
\frac{1}{N} \sum_{\vec{k}, \alpha, n} \frac{1}{1+e^{\beta\left(\epsilon_{\vec{k}, \alpha, n}-\lambda\right)}}=1
$$

The mean field amplitudes $t$ and $r$ are obtained by minimizing the free energy at a given temperature. As $T$ is increased from zero, we find that the $F$ vs. $r$ curve is qualitatively identical to the $E$ vs. $r$ curve at $T=0$ (Fig.(2)). Thus, the disconnected plaquette state $(r=$ 1) remains as the lowest free energy state (note that since $r=1$ is trivially a self-consistent solution, it is always a free energy extremum). Meanwhile the short-ranged correlation $t$ decreases as $T$ increases. In Fig.(3) we show the values of $t$ that minimizes the free energy for $r=0$ and $r=1$ as a function of $T$. Other $r$ value curves lie between these two curves. We see that at critical temperature $T_{t}=0.75, t \rightarrow 0$ for all values of $r$. Above $T_{t}$, even nearest neighbor sites are uncorrelated. $T_{t}$ can be calculated analytically easily. The free energy $F_{M F}$ for $H_{M F}$ in eq.(17) can be formally expanded in a series in $t_{i j}$. Assuming the transition in $t$ is continuous (which is likely since $F_{M F}$ is even in $t$ ), $T_{t}$ is given by the temperature where the coefficient in front of the quadratic term changes sign from positive to negative. To quadratic order, $F_{M F}$ can be easily found using perturbation by taking the chemical potential part of $H_{M F}$ as the unperturbed Hamiltonian $H_{0}$ and the remaining part as the perturbation $H^{\prime}$ (essentially doing high temperature expansion in $H^{\prime}$ ). By expanding to second order in $H^{\prime}$, we obtain

$$
F_{M F}=F_{0}+\sum_{\langle i j\rangle}\left(1-\frac{3}{4} \beta\right) t_{i j}^{2}+O\left(t^{4}\right) .
$$

From this, we see that $T_{t}=3 / 4$ and that $T_{t}$ is independent of the configuration of $t_{i j}$, hence of $r$. The best configuration for $t_{i j}$ is determined by the higher order terms in $F_{M F}$. Our 
calculation indicates it corresponds to $r=1$. Since for all $T<T_{t}, r=1$, the transition in $r$ is preempted by the transition in $t$. Within our MFT, we are therefore not able to answer the question of whether the plaquette ordering transition is a first order or a continuous one. However, the mean field transition in $t$ is unphysical and merely signifies a crossover from weak to strong nearest neighbor correlations. We thus interpret our mean field result to imply that as soon as significant nearest neighbor correlations develop, the system has complete plaquette ordering.

We now turn to the triangular lattice case. Similar to the square lattice, we consider plaquette ordering due to spontaneous formation of different (type-A and type-B) bond amplitudes as shown in Fig.(1). On the triangular lattice, there are more type-B bonds than A-type. Out of $3 \mathrm{~N}$ bonds, 5N/4 are A-type, and 7N/4 are B-type. Rigorously speaking, the bonds within A-type (or B-type) are not all equivalent. For example, among the A-type bonds illustrated in Fig.(1), the diagonal one has different connectivity from the four edge bonds. This requires, in principle, to assign different amplitudes for these bonds in a fully self-consistent mean field theory. Instead, we approximate all A bonds and all B bonds to be identical among themselves, which simplifies the calculation greatly. Our calculation then amounts to a variational calculation with two types of bonds or equivalently, a mean field calculation that is self-consistent when averaged over the A bonds or over the B bonds. The physics should not be affected qualitatively by this simplification. Quantitatively, we have also checked this point by examining a single A-plaquette problem explicitly. Assuming all bonds to be equivalent, we find $t_{a}=1.025$, while allowing the 4 edge bonds $\left(t_{a}^{\prime}\right)$ and the diagonal bond $\left(t_{a}^{\prime \prime}\right)$ to be different, we find $t_{a}^{\prime}=0.943$ and $t_{a}^{\prime \prime}=1.333$, giving an average of 1.021. The ground state energies in the two cases are also within $2 \%$ of each other, with values -1.31 and -1.33 respectively. The simplified scheme of only allowing two different bond strengths is therefore sufficient for our purpose.

We thus introduce two average mean field amplitudes: one for A-type and one for Btype. The self-consistent condition is then given by their average one, $t_{a}=\sum\left\langle\Phi_{i j}\right\rangle / N_{A}$, and $t_{b}=\sum\left\langle\Phi_{i j}\right\rangle / N_{B}$, where $\langle i, j\rangle \in\{A\}$ and $\in\{B\}$ respectively, and $N_{A}$ and $N_{B}$ are the 
number of $\mathrm{A}$ and $\mathrm{B}$ type bonds. With this approximation, the mean field Hamiltonian in a triangular lattice reads,

$$
\begin{aligned}
H_{m f}= & -\sum_{\vec{k}, n}\left[t\left(\cos k_{x} a+\cos k_{y} a\right)+(1-r / 2) t \cos \left(k_{x} a+k_{y} a\right)\right] c_{\vec{k}, n}^{\dagger} c_{\vec{k}, n} \\
& +i r t \sum_{\vec{k}, n}\left(\sin k_{x} a+\frac{1}{2} \sin \left(k_{x} a+k_{y} a\right)\right) c_{\vec{k}+(\pi, 0), n}^{\dagger} c_{\vec{k}, n} \\
& +i r t \sum_{\vec{k}, n}\left(\sin k_{y} a+\frac{1}{2} \sin \left(k_{x} a+k_{y} a\right)\right) c_{\vec{k}+(0, \pi), n}^{\dagger} c_{\vec{k}, n} \\
& -r t \sum_{\vec{k}, n} \frac{1}{2} \cos \left(k_{x} a+k_{y} a\right) c_{\vec{k}+(\pi, \pi), n}^{\dagger} c_{\vec{k}, n} \\
& +\frac{N t^{2}}{4}\left(3+3 r^{2}-r\right)-\lambda\left(\sum_{\vec{k}, n} c_{\vec{k}, n}^{\dagger} c_{\vec{k}, n}-N\right) .
\end{aligned}
$$

In the above eqn., we have used the same notation as for the square lattice. Note that in the triangular lattice, there is a lack of symmetry between the strong and the weak bonds. Consequently, the Hamiltonian does not possess all the symmetries of the square lattice. In particular, the system is not symmetric under the transformation $r \rightarrow-r$. From the definition of $r$ and $t$, we have a symmetry in parameter space, $r \rightarrow 1 / r, t \rightarrow r t$. This allows us to study $r$ within $[-1,1] . t \rightarrow-t$ symmetry is also not present for the triangular lattice. For $r=0$ and $r=1$, the energy is minimized by $t$ positive, and we assume this holds for other $r \neq 0$ also. The mean field Hamiltonian can be written in the diagonalized form,

$$
H_{m f}=\sum_{\vec{k}, \alpha, n} \epsilon_{\vec{k}, \alpha, n} \beta_{\vec{k}, \alpha, n}^{\dagger} \beta_{\vec{k}, \alpha, n}+\frac{N t^{2}}{4}\left[3\left(1+r^{2}\right)-r\right]-\lambda\left(\sum_{\vec{k}, \alpha, n} \beta_{\vec{k}, \alpha, n}^{\dagger} \beta_{\vec{k}, \alpha, n}-N\right) .
$$

The ground state is given by a filled Fermi sea, whose total energy is given by

$$
\frac{E}{N}=\frac{1}{N} \sum_{\vec{k}, \alpha, n} \epsilon_{\vec{k}, \alpha, n} \theta\left(\lambda-\epsilon_{\vec{k}, \alpha, n}\right)+\frac{t^{2}}{4}\left[3\left(1+r^{2}\right)-r\right]
$$

Since there is no $r \rightarrow-r$ symmetry, $E(r) \neq E(-r)$. Our mean field result shows that $E(r)$ has lower energy for positive $r$. Similar to the square lattice, the ground state is the disconnected plaquette state corresponding to $r=1$. In this case, the ground state is 
12-fold degenerate. Again, the local stability of $r=1$ can be confirmed by second order perturbation theory. This also gives a heuristic understanding of why the energy is lower for positive vs. negative $r$. The $r=-1$ state is a plaquette state such that neighboring plaquettes are connected by a single diagonal bond of equal strength as the bonds in the plaquette. Now imagine this connecting bond is not equal but much weaker, which will make this state quite similar to $r$ less than but very close to 1 . The local stability of $r=1$ is thus consistent with it being lower in energy than $r=-1$, and by continuity with positive $r$ having the lower energy vs. negative $r$ for at least a range of $r$ close to 1 .

To study the finite temperature phase, we minimize the free energy

$$
\begin{gathered}
\frac{F}{N}=-\frac{k_{b} T}{N} \sum_{\vec{k}, \alpha, n} \ln \left[1+e^{-\beta\left(\epsilon_{\vec{k}, \alpha, n}-\lambda\right)}\right]+\lambda+\frac{t^{2}}{4}\left[3\left(1+r^{2}\right)-r\right], \\
\frac{1}{N} \sum_{\vec{k}, \alpha, n} \frac{1}{1+e^{\beta\left(\epsilon_{\vec{k}, \alpha, n}-\lambda\right)}}=1 .
\end{gathered}
$$

As in the square lattice, $r=1$ remains the stable state at low $T$ (Fig.(团). On the triangular lattice however, the transition in $r$ is not preempted by the one in $t$, and we are able to study its phase transition. At $T \approx 0.74$, the curvature of the free energy curve at $r=0$ changes sign and $r=0$ changes from a local maximum to local minimum. When $T$ is increased to $T_{c}=0.782, r=0$ and $r=1$ states become degenerate in free energy and there is a first order transition from the disconnected plaquette state to the uniform state (or the other way round if we consider lowering the temperature). These results are summarized in Fig(5)). Since the lack of reflection symmetry in $r$ allows for a cubic invariant in the Landau theory for its phase transition, the first order transition obtained is to be expected. Indeed, the behavior of $F$ vs. $r$ as a function of $T$ is precisely what one would expect from such a Landau-Ginzburg free-energy functional. Upon further raising the temperature, the average nearest neighbor correlation continues to decrease, with the mean field transition to a completely uncorrelated state occurring at $T_{t} \approx 0.8$. Unlike the square lattice case, on the triangular lattice, the transition in $t$ occurs at different temperature for different $r$, i.e. $T_{t}=T_{t}(r)$. This can be readily understood from the lack of $t \rightarrow-t$ symmetry. As a 
result, the corresponding $F_{M F}($ eq.(7) ) contains odd power terms, which in turn results in first order transition in $t_{i j}$. Naturally then, the transition temperature will occur above the second order temperature at 0.75 and will depend on the configuration of $t_{i j}$, hence on $r$. As always, the transition in $t$ should be interpreted physically as a crossover.

In summary, within MFT, we find the uniform mean field state to be unstable with respect to plaquette ordering at $T=0$ for both the square and triangular lattices. For the triangular lattice, the plaquette ordering transition at finite temperature is first order, in agreement with Landau theory argument. For the square lattice, the plaquette to uniform state is preempted by a mean field transition in $t$, and MFT does not give an unambiguous answer to if the plaquette transition is first order or continuous. Taking the MFT on its face value, the transition is continuous as there is no entropy jump, although this is complicated by it being also a transition in $t$. Based on the reflection symmetry in $r$, Landau theory would also suggest a continuous transition. However, we cannot rule out a first order transition due to a negative quartic invariant in the Landau free energy functional or due to fluctuations. For the triangular lattice, the plaquette transition is first order as expected from symmetry.

Within MFT, the ground states of the SU(4) model in both square and triangular lattice are found to be disconnected plaquette states $(r=1)$. Naively, this implies huge degeneracies in the ground states. Consider a lattice with periodic boundary condition. All the plaquette states with plaquettes covering all the lattice sites have the same lowest mean field energy. A simple lower bound for ground state degeneracy would then be $D \approx 2^{\sqrt{N}}$ for the square lattice, and $D \approx 3^{N / 12}$ for the triangular lattice. However, such plaquette states are not orthogonal to each other whether we consider the mean field states or the actual SU(4) states. They may not even be independent, so these lower bounds for degeneracy may not be valid, but should be taken as suggestive of huge degeneracy. Obviously, the exact SU(4) ground state will not exhibit saturated plaquette ordering (there will be for example resonance between plaquette states), and the actual ground state will only be 4 -fold and 12-fold degenerate respectively. Nevertheless, we may still interpret the mean field ground state to imply a very strong, although not saturated plaquette ordering, so that plaquettes 
are only weakly coupled. We would then expect a huge number of low lying energy states in the energy spectrum. This is consistent with the preliminary results of Penc et al. [20] on the square and triangular lattices using variational calculations on relatively large systems. This work was supported in part by DOE grant No. DE/FG03-01ER45687 and by URC summer fellowship at University of Cincinnati. The authors wish to thank F. Mila and X. R. Wang for discussions. 


\section{REFERENCES}

[1] Y. Tokura and N. Nagaosa, Science 288, 462 (2000).

[2] K.I. Kugel' and D.I. Khomskii, Fiz. Nizk. Temp. 6, 207 (1980) [Sov. J. Low Temp. Phys. 6, $99(1980)]$.

[3] Y.Q. Li, Michael Ma, D.N. Shi, and F. C. Zhang, Phys. Rev. Lett. 81, 3527 (1998).

[4] D.B. McWhan, T.M. Rice, and J.P. Remeika, Phy. Rev. Lett 23, 1384(1969); D.B. McWhan, A. Menth, J.P. Remeika, W.F. Brinkman, and T.M. Rice, Phys. Rev. B 7, 1920(1973).

[5] C. Castellani, C.R. Natoli, and J. Ranninger, Phys. Rev. B18, 4945, 4967 and 5001 (1978).

[6] W. Bao, C. Broholm, S.A. Carter, T.F. Rosenbaum, G. Apeli, S.F. Trevino, P. Metcalf, J.M. Honig, and J. Spalek, Phys. Rev. : Lett. 71, 766 (1993).

[7] J.H. Park, L.H. Tjeng, A. Tanaka, J.W. Allen, C.T. Chen, P. Metcalf, J.M. Honig, F.M.F. de Groot, and G.A. Sawatzky, Phys. Rev.61, 11506 (2000).

[8] F. Mila, R. Shiina, F. C. Zhang, A. Joshi, M. Ma, V. Anisimov, and T. M. Rice, Phys. Rev. Lett. 85, 1714 (2000); A. Joshi, Michael Ma, F. C. Zhang, Phys. Rev. Lett. 86, 5743 (2001); R. Shiina, F. Mila, F.-C. Zhang, and T.M. Rice, Phys. Rev. B 63, 144422 (2001).

[9] K. Yamaura, M. Takano, A. Hirano, and R. Kanno, J. Solid State Chem. 127, 109 (1997), and references therein.

[10] Y. Kataoka et al. J. Phys. Soc. Jpn. 67, 3703 (1998).

[11] F. Reynaud, D. Mertz, F. Celestini, J.-M. Debierre, A. M. Ghorayeb, P. Simon, A. Stepanov, J. Voiron, and C. Delmas, Phys. Rev. Lett. 86, 3638 (2001).

[12] L. F. Feiner, A. M. Oles, and J. Zaanen, Phys. Rev. Lett. 78, 2799 (1997). 
[13] Y. Murakami, H. Kawada, H. Kawata, M. Tanaka, T. Arima, Y. Moritomo, and Y. Tokura, Phys. Rev. Lett. 80, 1932 (1998).

[14] L. Paolasini, C. Vettier, F. de Bergevin, F. Yakhou, D. Mannix, A. Stunault, W. Neubeck, M. Altarelli, M. Fabrizio, P. A. Metcalf, and J.M. Honig, Phys. Rev. Lett. 82, 4719 (1999).

[15] D. P. Arovas and A. Auerbach, Phys. Rev. B 38, 316 (1988).

[16] Guang-Ming Zhang and Shun-Qing Shen, Phys. Rev. Lett. 87, 157201 (2001); condmat/0109234

[17] M. van den Bossche, F.C. Zhang, F. Mila, Eur. Phys. J.B17, 367 (2000).

[18] G. Baskaran, Z. Zou, and P. W. Anderson, Solid State Commun. 63, 973 (1987).

[19] I. Affleck and J. B. Marston, Phys. Rev. B 37, 3774 (1988).

[20] K. Penc, M. Mambrini, and F. Mila, private communication. 


\section{FIGURES}
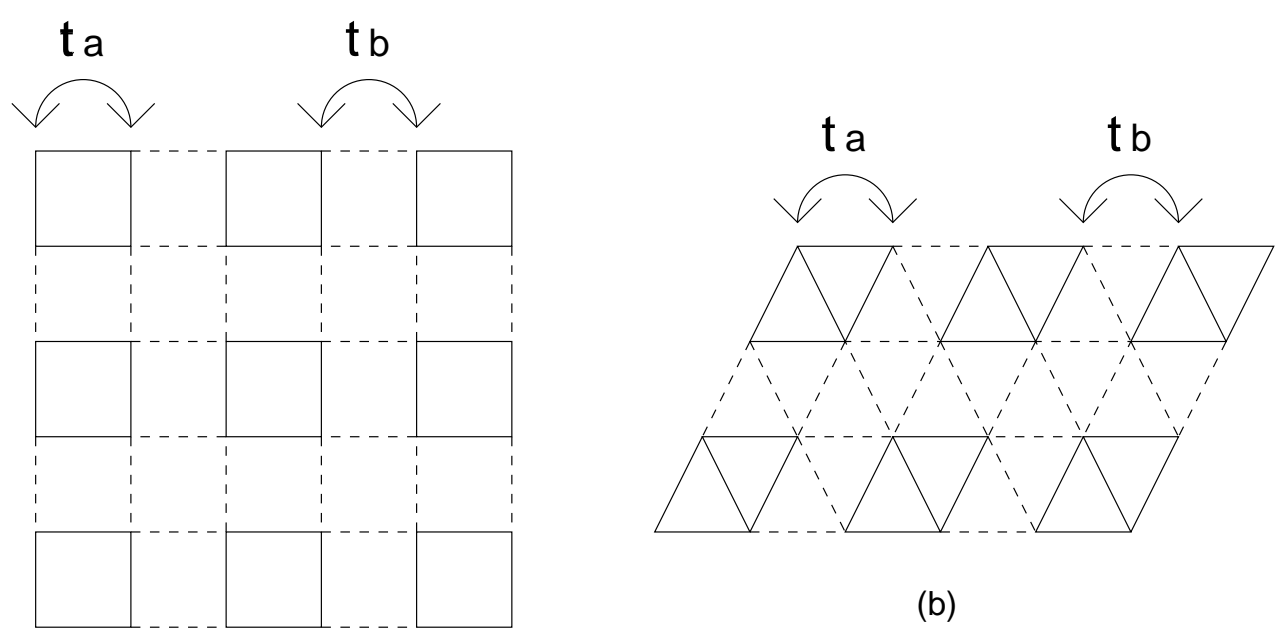

"A" type.

"B" type

(b)

(a)

FIG. 1. A schematic representation of plaquettes on a) square lattice, b) triangular lattice. Each solid line represents a strong bond connecting two lattice sites, with hopping amplitude $t_{a}$. Each dashed line represents a weak bond with hopping amplitude $t_{b}$.

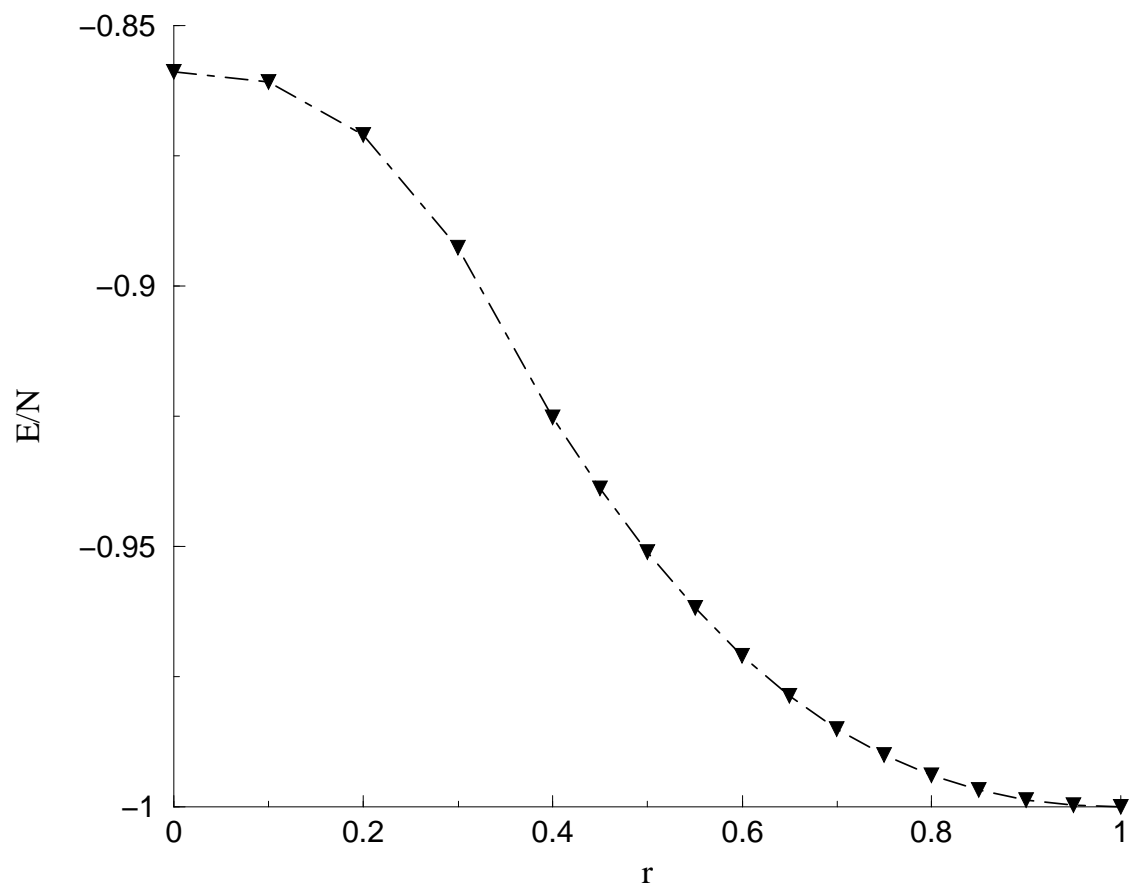


FIG. 2. Energy per site, $(E / N)$, in eqn.(6) is plotted as a function of $r$ for a square lattice. $E / N$ monotonically decreases as $r$ increases from 0 to 1 . The lowest energy state corresponds to $r=1$, the disconnected plaquette state.

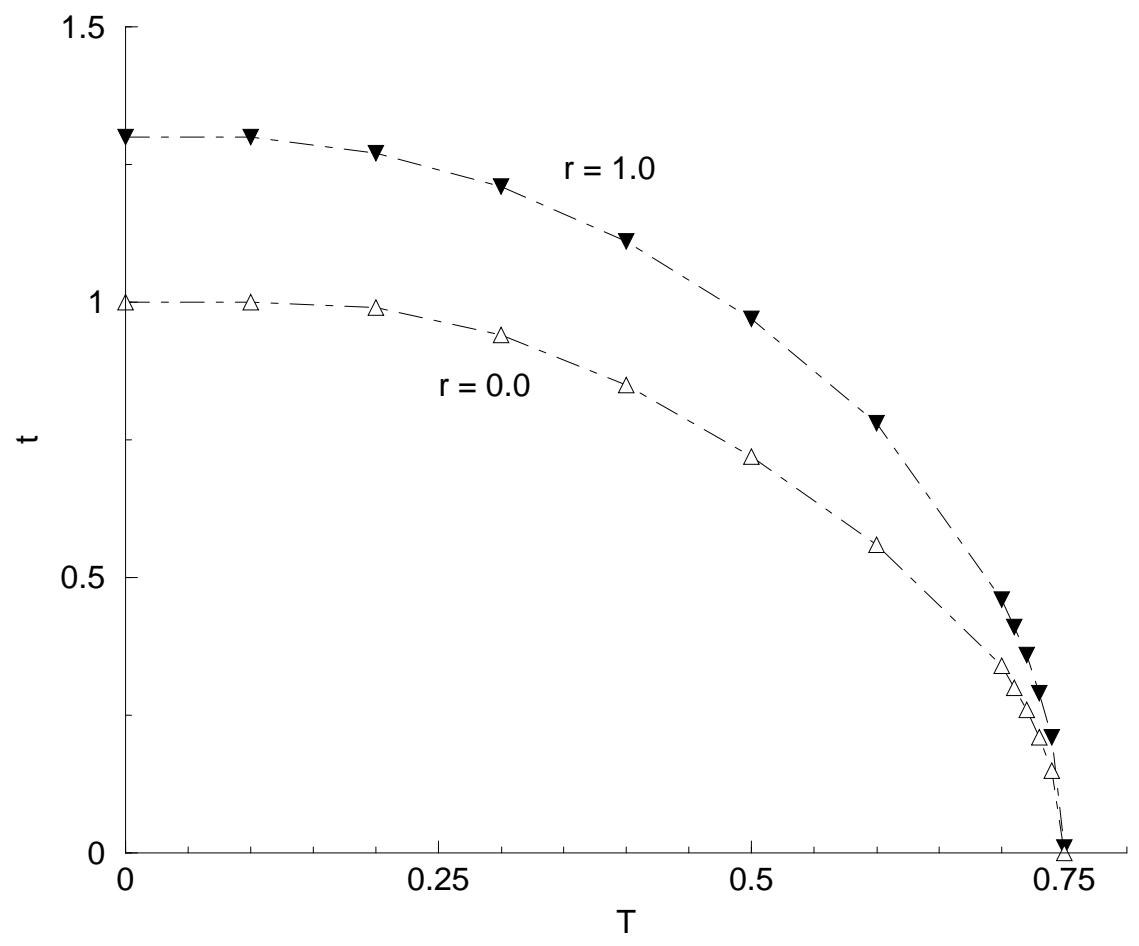

FIG. 3. The optimal values of $t$ as functions of temperature $T$ for $r=0$ and $r=1$ on a square lattice. For $0 \leq r \leq 1, t$ v.s. $T$ curves lie between these two curves. The short range correlation between neighboring lattice sites, characterized by $t$, decreases with increasing $T$. At $T=0.75$, $t \rightarrow 0$ for all values of $r$. 


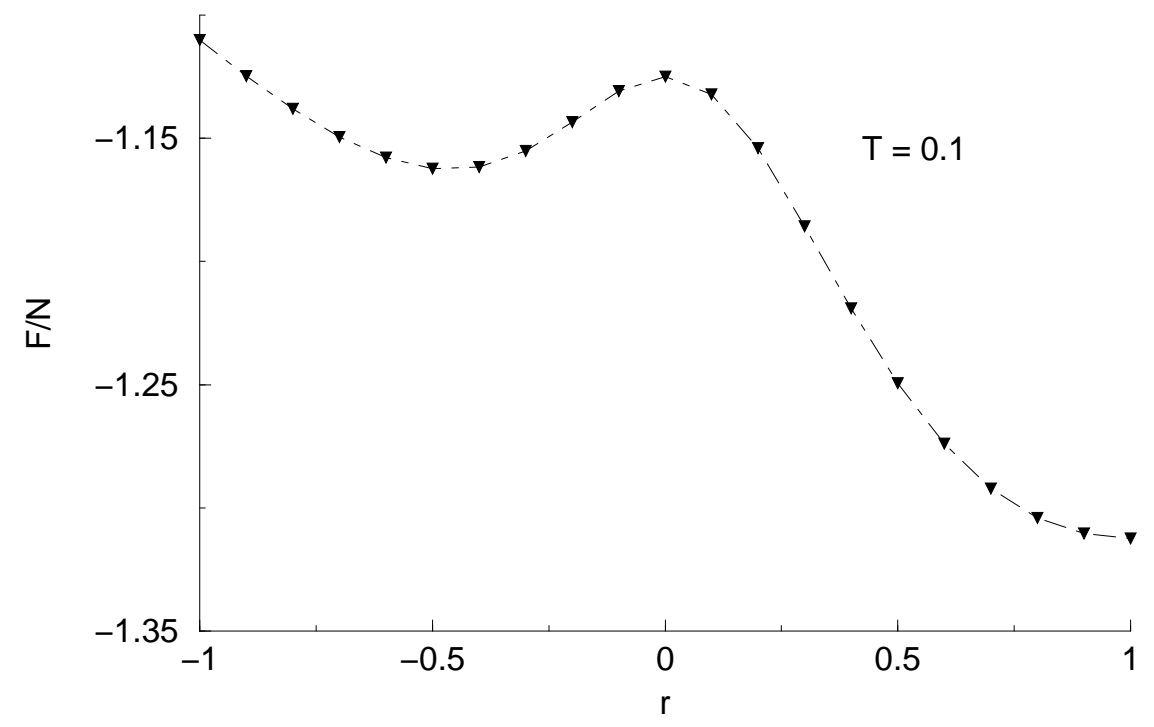

FIG. 4. Free energy per site from eqn.(10) is plotted with respect to $r$ for the triangular lattice case at $T=0.1$. At this temperature, $r=1$ remains the stable state and $r=0$ remains as a local maximum.
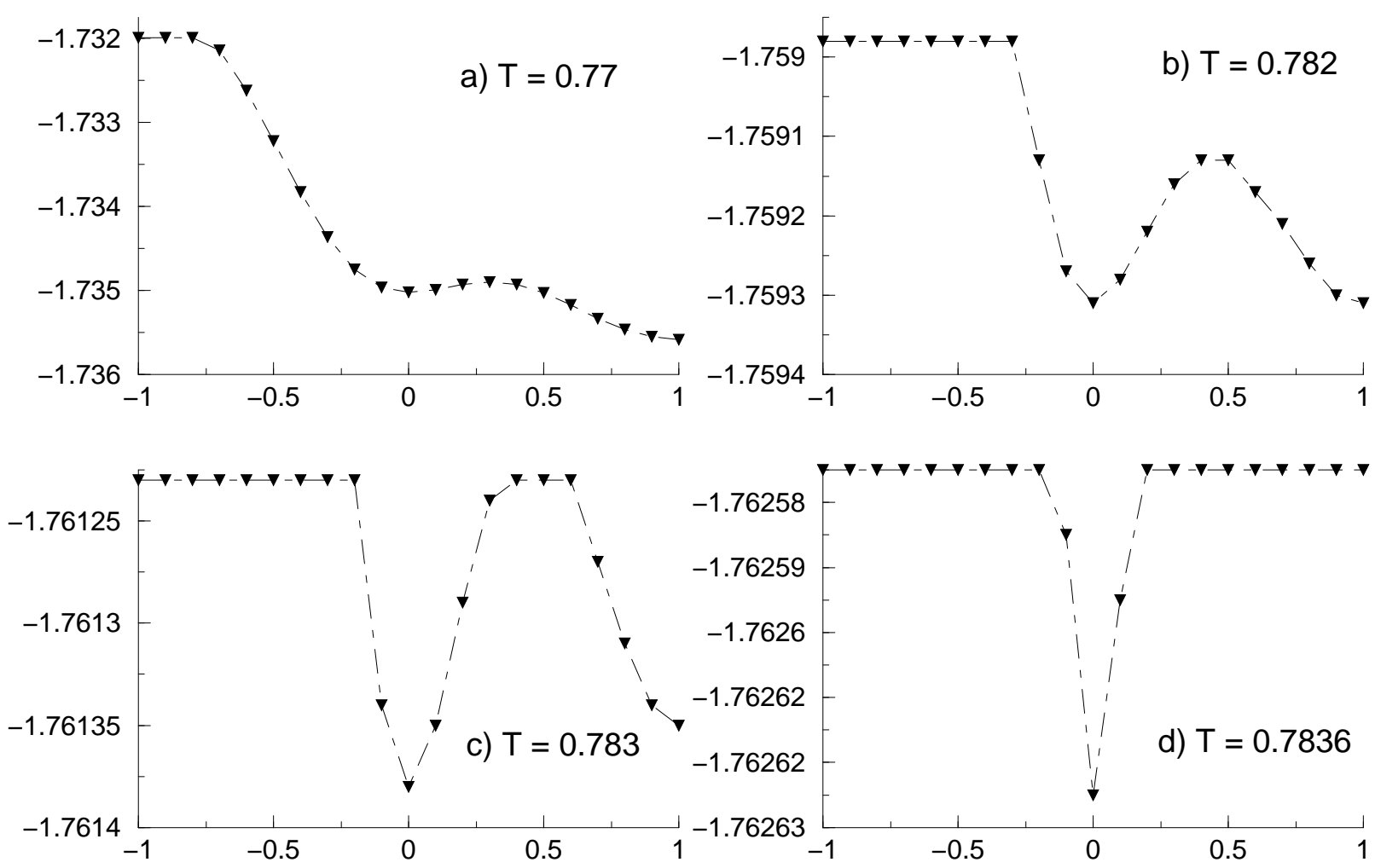
FIG. 5. Free energy per site v.s. $r$ on the triangular lattice. a) For $0.74<T<0.782, r=1$ is the lowest free energy state while $r=0$ is locally stable. b) At $T=0.782, r=0$ and $r=1$ become degenerate. At this $\mathrm{T}$, the transition in $t$ for a given $r$ has already occurred for a range of $r<0$. c) At $\mathrm{T}=0.783, r=0$ is the stable state and $r=1$ is a local minimum. Transition in $t$ begins to occur for some $r>0$. d) At $T=0.7836$, the transition in $t$ has occurred for $r=1$, leaving $r=0$ as the only minimum. 